\title{
Relations entre la lumière et la photosynthèse du phytoplanc- ton dans un réservoir mésotrophe (Pareloup) : variations sai- sonnières des paramètres
}

\author{
J. Capblancq ${ }^{1}$ \\ J.M. Thébault 1 \\ A. Jrad ${ }^{1}$
}

Mots clés : Lacs, phytoplancton, production primaire, relations photosynthèse-lumière, modèles de production.

Les relations entre les profils verticaux de production photosynthétique du phytoplancton et la lumière transmise ont été analysées sur une série de 48 expériences réalisées sur la retenue de Pareloup. Le taux de photosynthèse spécifique à saturation par la lumière $\left(\mathrm{P}_{\mathrm{opt}}\right)$ ) la pente initiale de la courbe photosynthèse-lumière $(\alpha)$ et le rendement quantique global en éclairement limitant $\left(\phi_{\mathrm{m}}\right)$ varient de plus d'un ordre de grandeur. Une forte corrélation entre $P_{o p t}$ et $\alpha$ a été mise en évidence qui permet d'envisager la possibilité d'estimer l'une des valeurs à partir de l'autre. Ces deux paramètres sont corrélés avec la température et varient inversement aux concentrations de chlorophylle mais ces deux facteurs n'expliquent que $43 \%$ de la variance $P_{o p t}$. Des modifications de la réponse photosynthétique du phytoplancton ont été observées en été lorsque la zone euphotique se stratifie. Ces résultats sont discutés par rapport aux conceptions récentes sur l'écophysiologie des algues planctoniques et aux modèles de production primaire du phytoplancton.

Relationships between phytoplankton photosynthesis and light in a mesotrophic reservoir (Pareloup) : seasonal variations of parameters.

Keywords : Lakes, phytoplankton, primary production, photosynthesis-light relationship, models of production.

The relationships of vertical profiles of phytoplankton photosynthesis to underwater light were analysed in a series of 48 experiments performed in the mesotrophic reservoir of Pareloup. The light saturated photosynthetic rates $\left(\mathrm{P}_{\mathrm{Op}}\right)$, the initial slope of light saturation curves $(\alpha)$ and the quantum yield at limiting light intensities $(\phi \mathrm{m})$ vary over more than one order of magnitude. A strong correlation appears between $\alpha$ and $\mathrm{P}_{\mathrm{Opt}}$ which suggest the possibility of predicting the values of one from those of the other. Both parameters are correlated with chlorophyll concentrations and temperature but these two factors account for only $43 \%$ of the variance of $P_{\text {opt }}$. Changes in the photosynthetic light responses by phytoplank ton were observed during summer when the euphotic layer became thermally stratified. These results are discussed with regard to predictive models of phytoplankton production.

\section{Introduction}

Les modèles de production du phytoplancton visent à simuler les profils verticaux de photosynthèse afin de calculer la production primaire de la zone euphotique des lacs et des océans à partir de la mesure d'un nombre restreint de valeurs caractéristiques. Ces modèles reposent sur une

1. Laboratoire d'Hydrobiologie, UA 695 du C.N.R.S., Université Paul Sabatier, 118, route de Narbonne, 31062 Toulouse Cedex, France. formulation mathématique de la courbe de saturation qui décrit la variation du taux d'activité photosynthétique par unité de biomasse $(\mathbf{P})$ en fonction de l'intensité (x) de la lumière. Plusieurs auteurs (Talling 1957, Vollenweider 1965, Rodhe 1965, Bannister 1974, Jassby \& Platt 1976) montrent que la courbe $P=f(I)$ peut être correctement décrite à l'aide de deux paramètres : le taux de photosynthèse optimum à saturation par la lumière $\left(\mathrm{P}_{\mathrm{Opt}}\right)$ et la pente initiale de la courbe en éclairement limitant $(\alpha)$ ou par des valeurs qui leur sont associées $\left(I_{k}=\right.$ $\mathrm{P}_{\text {opt }} / \alpha=$ intensité seuil de saturation par la 
lumière: $\phi_{\mathrm{m}}=$ rendement quantique global de la photosynthèse en lumière limitante).

Il est généralement admis que $P_{\text {opı }}$ et $\alpha$ dépendent respectivement de l'intensité des processus enzymatiques et photochimiques de la photosynthèse. En exprimant ces paramètres par unité de chlorophylle (constituant variant cans les cellules selon les espèces, la taille, l'état nutritionnel, la préadaptation à l'ombre ou à la lumière), on peut s'attendre à des valeurs de $\alpha$ et $\phi_{\mathrm{m}}$ relativement constantes, $P_{\text {opr }}$ et $I_{k}$ variant essentiellement avec la température selon un $\mathrm{Q}_{10}$ voisin de 2 (Capblancq 1982). Cependant, des travaux plus récents (Coté $\&$ Platt 1983, Tilzer 1984, Fee \& al. 1987) indiquent que ces paramètres peuvent varier dans des limites assez. larges. Cette variabilité limite considérablement les possibilités d'utilisation des modèles de production.

Le travai] entrepris sur le réservoir de Pareloup avait pour objectifs (1) de définir la plage de variations des parametres de la courbe $P=f(I)$ au cours d'un cycle annuel et (2) de rechercher parmi les composantes du milieu celles qui sont susceptibles d'expliquer ces variations.

\section{Méthodes de mesure}

L'analyse est basée sur 48 profils de distribution verticale des taux de photosynthèse établis à une station centrale du lac de Pareloup entre juin 1983 et juin 1985 (35 profils) et d'avril à novembre 1987 (13 profils).

Les mesures ont été effectuées dans des flacons transparents et opaques d'une capacité de $125 \mathrm{~cm}^{3}$, immergés à différentes profondeurs pendant 2 heures au midi après ajout de $55 \mathrm{KBq}$ de ${ }^{14} \mathrm{CO}_{3} \mathrm{NaH}$ (CEA, Saclay). Des filtres d'une porosité de $0,45 \mu \mathrm{m}$ ont été utilisés pour filtrer les échantillons dès la fin de la période d'incubation. Après un rinçage avec $15 \mathrm{~cm}^{3}$ d'eau de lac préfiltrée, les filtres ont été immédiatement transférés dans des fioles contenant du liquide scintillant (Ready Solv HP/b, Beckman). La radioactivité des filtres et celle des solutions ont été mesurées par scintillation liquide et corrigées pour le quenching. La radioactivité des filtres correspondant aux flacons opaques a été soustraite de celle des flacons transparents. Les concentrations de ${ }^{12} \mathrm{CO}_{2}$ ont été calculées à partir des valeurs de $\mathrm{pH}$ et d'alcalinité.
Les pigments photosynthétiques ont été dosés par une méthode spectrophotométrique décrite par Marker \& al. (1980), en utilisant de l'éthanol porté à ébullition pour l'extraction. La D.O. de l'extrait a été mesurée avant et après acidification pour estimer la fraction de phéopigments.

L'éclairement incident a été calculé à partir des valeurs fournies par un pyranomètre couplé à un intégrateur en considérant que la fraction comprise entre 400 et $700 \mathrm{~nm}$ (PAR ou Photosynthetically Active Radiation) est égale à $46 \%$ du rayonnement total et que les pertes par réflexion à la surface sont de $10 \%$. Pour les mesures de l'atténuation de la lumière dans l'eau, nous avons utilisè une sonde immergeable (LI-192 S) reliée à un quantamètre (Lambda Instruments). Le coefficient d'atténuation vertical $\left(\epsilon_{v}\right)$ a été calculé par régression des valeurs en fonction de la profondeur.

\section{Analyse des données}

Les profils verticaux de production photosynthétique ont été analysés par référence aux modèles qui décrivent les variations en profondeur du taux de photosynthèse spécifique $\left(\mathrm{P}_{2}\right)$ en fonction de l'intensité de l'éclairement disponible $\left(\mathrm{I}_{\mathrm{z}}\right)$. Dans cette optique, nous avons utilisé les variables, les paramètres et les unités définis ci-après :

$\mathrm{CHL}=$ concentration en chlorophylle $; \mathrm{mg} \cdot \mathrm{m}^{-}-3$

$\mathbf{I}_{\circ}^{\prime}=$ intensité des PAR (400-700 nm) immédiatement sous la surface; W. - $^{-2}$ (= $4,6 \mu$ E.m- $\left.\mathrm{m}^{-1} \mathrm{~s}^{-1}\right)(=16,56 \mathrm{mE} \cdot \mathrm{m}-2 \mathrm{~h}-1)$ (16,56 mmol.m-2h-1)

$I_{z}=$ intensité des PAR à la profondeur $z$; mêmes unités

$\epsilon \mathrm{v}=$ coefficient moyen d'atténuation verticale de la lumière ; $\mathrm{m}^{-1}$

$\mathbf{Z}_{\text {od }}=$ profondeur optique $=$ épaisseur en mètres d'une couche d'eau absorbant $50 \%$ de la lumière

$\epsilon_{\mathrm{c}}=$ coefficient d'atténuation spécifique de la chlorophylle; $\mathrm{m}^{-1}$ ! (mg CHL.m-3)- 1

CHL $_{\varepsilon} \mathrm{c}^{\prime \epsilon} \mathrm{v}=$ fraction du coefficient d'at ténuation dủ aux algues

$\mathrm{I}_{\mathrm{abs}}=$ quantité de lumière absorbée par la chlorophylle dans une couche d'eau d'épaisseur $\Delta z$, $=\mathrm{I}_{\mathrm{z}-\Delta z \cdot\left(1-\mathrm{e}^{-\epsilon} \mathrm{v} \cdot \Delta z\right)} \cdot($ CHL.E $\mathrm{d} / \epsilon \mathrm{v})$ 
$P_{z}=$ taux de photosynthèse spécifique à la profondeur $\mathrm{z}$; mg C. (mg CHL) $)^{-1 \mathrm{~h}^{-1}}$

$\mathrm{P}_{\mathrm{opt}}=$ taux de photosynthèse spécifique optimum ; mêmes unités

$\mathbf{I}_{\mathrm{k}}=$ intensité seuil de saturation; mêmes unitês que pour $\mathrm{I}_{\mathrm{o}}$

$\alpha=$ pente initiale de la courbe $\mathbf{P}=f(\mathrm{I})$ pour $I \leqslant 0,5 \mathbf{I}_{\mathrm{k}} ; \mathrm{mgC}$. (mg CHL. mE PAR)- $1 \mathrm{~m}^{2}$

$\phi_{\mathrm{z}}=$ rendement quantique global de la photosyn. thèse à la profondeur $z$

= moles de carbone assimilé / moles de photons absorbés

$=\mathbf{P}_{\mathbf{z}} \cdot \mathbf{C H L} / \mathbf{I}_{\mathrm{abs}}$

$=P_{2} / 12 . I_{Z} \cdot f$ lorsque $\Delta z$ est très petit.

En lumière limitante $\left(I<I_{k}\right), P_{z}$ varie proportionnellement à $I_{z}$ et $\phi_{z}$ tend vers une valeur constante $\left(\phi_{\mathrm{m}}\right)$. En lumière saturante, $\mathrm{P}_{\mathrm{z}}$ atteint un maximum $\left(P_{\text {opt }}\right)$ et $\phi_{z}$ diminue lorsque $I_{z}$ augmente. Au point de transition $I_{k}$ la relation s'écrit (Tilzer 1984) :

$$
\begin{array}{ll}
\text { d'où } & \mathrm{I}_{\mathrm{k}}=\mathrm{P}_{\mathrm{opt}} / 12 \epsilon_{\mathrm{c}} \cdot \phi_{\mathrm{m}} \\
\text { et puisque } & \alpha=\mathrm{P}_{\mathrm{opt}} / \mathrm{I}_{\mathrm{k}} \\
& \alpha=12 \epsilon_{\mathrm{c}} \cdot \phi_{\mathrm{m}}
\end{array}
$$

\section{Evolution saisonnière des compo- santes climatiques et du peuplement phytoplanctonique}

Située au sud-ouest du Massif Central à une altitude de 850 mètres, la retenue hydroélectrique de Pareloup (superficie $=13,5 \mathrm{~km}^{2}$; profondeur moyenne $=12,5 \mathrm{~m}$ ) est alimentée par les apports naturels du bassin versant du Vioulou (superficie $=122 \mathrm{~km}^{2}$ ) et par les eaux pompées dans le bassin du Viaur.

Son régime thermique (fig. 1) se caractérise par une stratification de la masse d'eau entre la fin juin et la fin septembre. Les poussées de phytoplancton se produisent lors des périodes d'isothermie de printemps - le peuplement est alors composé de Diatomées avec une très forte dominance de Asterionella formosa - et d'érosion de la thermocline en automne. La biomasse diminue fortement durant la période de stratification; elle est composée de Cyanophytes (Microcystis, Anabaena, Merismopedia), de Pyrrophytes (Ceratium, Cryptomonas, Rhodomo. nas) et de Chlorophytes (Crucigenia, Pediastrum,
Ankistrodesmus, Gloeocystis) dans des proportions variables d'une année à l'autre. Les Diatomées (Melosira ambigua $+A$. formosa réapparaissent lors de la poussée automnale.

La distribution verticale du phytoplancton est homogène en période d'isothermie. Durant l'été, deux situations différentes ont été rencontrées au cours de cette étude (fig. 1):

- en 1983 et 1984, le gradient de température qui apparaît dans la zane euphotique début juin évolue très rapidement; à partir du 15 juillet, la couche euphotique est pratiquement isotherme. Ces conditions se traduisent par des profils verticaux de distribution de la biomasse de phytoplancton le plus souvent uniformes.

- en 1987, la cauche euphotique reste stratifiée pendant tout l'été : cette stratification entraine la formation d'un maximum de chlorophylle au niveau du métalimnion qui correspond à la fois à une différenciation du peuplement (développement d'une population de Gonyostomum, Chloromonadophyte de grande taille, dans les niveaux inférieurs de la couche euphotique à partir du mois de juillet) et à un enrichissement des algues en chlorophylle (la teneur moyenne par cellule ou par unité de biomasse est 2 à 6 fois plus élevée entre 7 et 10 mètres qu'en surface). Les conditions météorologiques de la fin du mois d'août entraînent une dégradation de ces structures qui se reforment au cours du mois de septembre.

La profondeur de la zone euphotique $\left(Z_{e u}\right)$ varie, en relation avec le développement du phytoplancton, entre 5,5 et 12,5 mètres ( $f \mathrm{ig}$. 1). Les équations de régression entre $\epsilon_{v}$ et la concentration moyenne en chlorophylle, calculées sur les périodes juin 83 - juin 85 et mars-octobre 87 sont respectivement :

$$
\begin{aligned}
& \epsilon_{v}=0,448+0,021 \mathrm{CHL} \quad(\mathrm{r}=0,75 ; \mathrm{DL}=34) \\
& \epsilon_{v}=0,352+0,019 \mathrm{CHL} \quad(\mathrm{r}=0,81 ; \mathrm{DL}=30)
\end{aligned}
$$

Ces relations permettent d'estimer $\epsilon_{c}$ à $0,02 \mathrm{mg}^{-1} \mathrm{CHL} . \mathrm{m}^{2}$, valeur conforme à celles relevées dans la litterature. La fraction du coefficient d'extinction attribuable au phytoplancton $\left(\epsilon_{\mathrm{C}} \cdot \mathrm{CHL}\right.$ $/ \epsilon_{\mathrm{v}}$ ) est de 0,18 en moyenne ; elle varie entre 0,12 et $0,51(\bar{x}=0,24)$ au printemps et à l'automne, entre 0,04 et $0,21(\bar{x}=0,09)$ en été. 


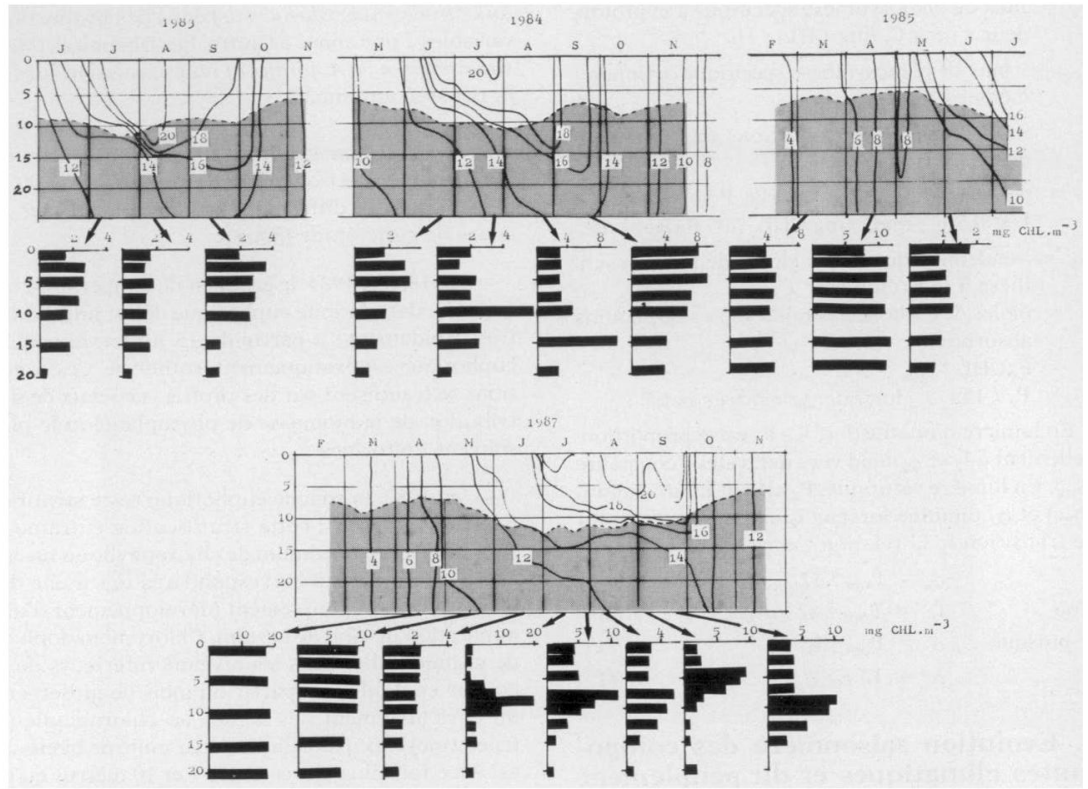

Fig. 1. Courbes isothermes, profondeur de la couche euphotique (en blanc) et exemples de profils de répartition verticale des concentrations en chlorophylle dans le lac de Pareloup.

\section{Profils verticaux de photosynthèse}

Les courbes obtenues en portant les taux d'activité spécifiques $\left(\mathrm{P}_{z}\right)$ sur une échelle de profondeurs graduée en profondeurs optiques $\left(Z_{o d}\right)$ sont de deux types :

- le premier, généralement observé de juin 1983 à juin 1985 ( $f$ ig. 2A, 2B, 2C) correspond à une faible stabilité thermique de la zone euphotique qui conduit à une distribution homogène du phytoplancton. Ces profils sont conformes au modele décrit par Rodhe (1965) : $P_{z}$ passe par un maximum $\left(P_{\text {opt }}\right)$ situé entre 0,5 et 2,5 metres selon la valeur de $I_{0}$ et décroit parallèlement à la lumière disponible pour $\mathbf{I}_{\mathbf{z}} \leqslant 0,5 \mathbf{I}_{\mathrm{k}}$. On note cependant que certains profils s'écartent de ce modèle, le plus souvent parce que
$P_{z}$ décroit plus vite que $I_{z}$ (profils du 26.03.85, du 11.09.84 ou du 28.09 .84 p. ex.).

- le second (fig. 2D) correspond à la formation de gradients de densité et de concentrations de pigments observés durant l'été 1987 . En règle générale, la diminution $P_{7}$ est plus forte que celle de $I_{z}$; elle est parfois très brutale (profils du 30.06 .87 et du 22.07.87). L'évolution des profils entre la fin mai et la fin juillet permet d'interpréter les variations de $\mathrm{P}_{\mathrm{z}}$ comme le résultat d'une adaptation progressive du phytoplancton des couches superficielles et profondes à des conditions moyennes d'éclairement différentes. L'adaptation aux faibles éclairements entraîne une augmentation de la teneur des algues en pigments mais une baisse du taux de photosynthèse par unité de chlorophylle. Ce même processus 

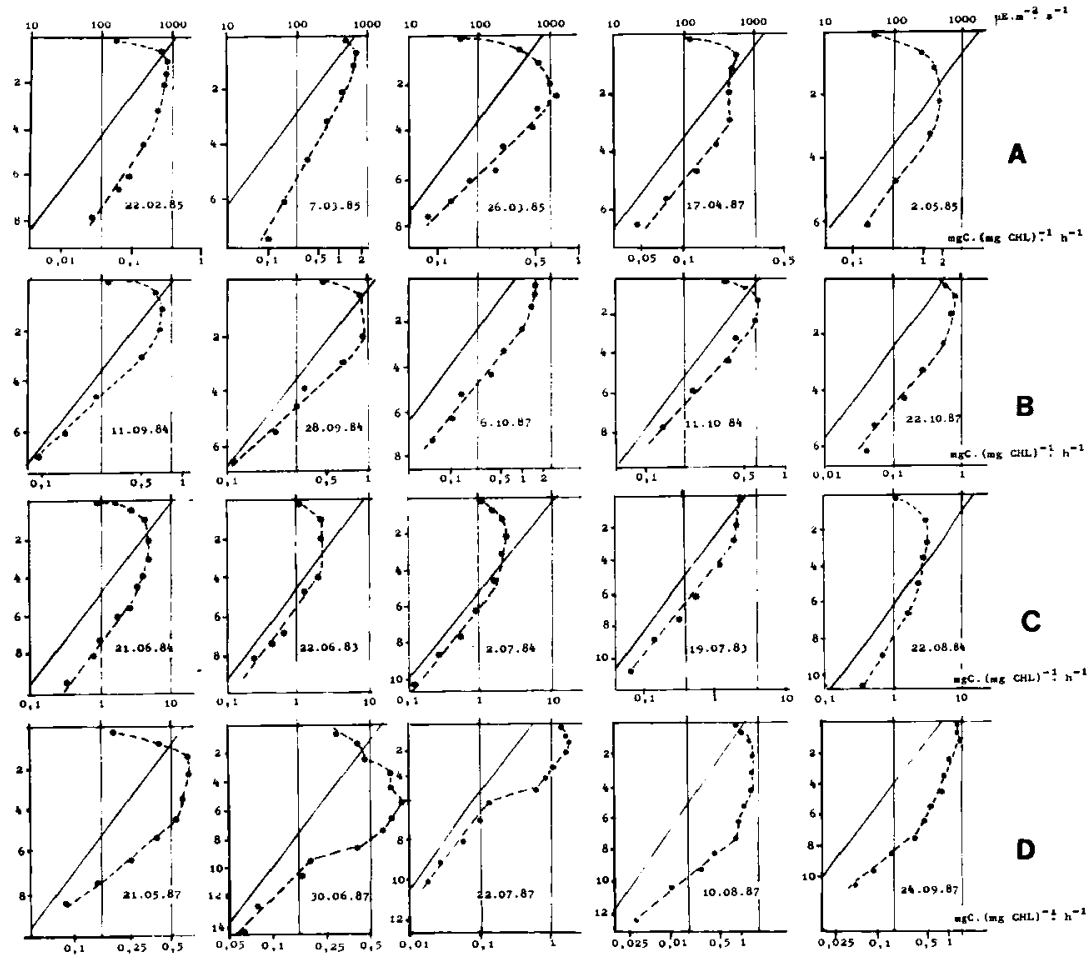

Fig. 2. Profils typiques du taux de photosynthèse spécifique (-*-) et d'intensité lumineuse (_-) en période d'isothermie de printemps $(A)$, d'automne $(B)$, de faible stabilité thermique en été $(C)$ et en présence d'un fort gradient thermique (D). Les valeurs de $I_{7}$ et de $P_{t}$ (échelles logarithmiques) sont figurées sur une échelle de profondeurs graduée en unités de profondeur optique $\left(Z_{\text {od }}\right)$. 


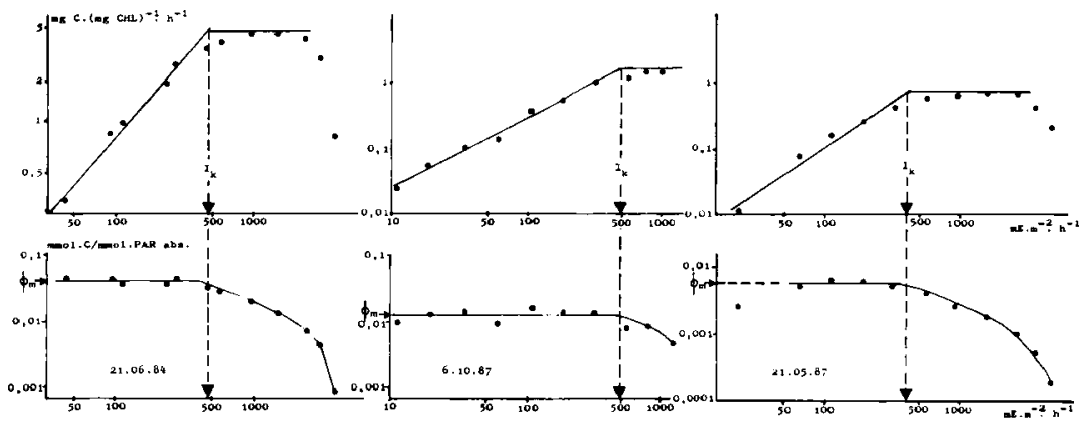

Fig. 3. Courbes illustrant la relation entre les taux de photosynthèse spécifique (en haut), le rendement quantique global ( $\phi$, en bas) et l'intensité de la lumière.

d'adaptation consécutif à de courtes périodes de stabilité de la couche euphotique pourrait expliquer les divergences observées entre $P_{z}$ et $I_{z}$ dans des conditions d'apparente homogénéité.

\section{Variations des paramètres de la courbe $P=f(I)$}

Les relations, formulées au § 3, entre les paramètres de la courbe $P=f(I)$ sont illustrées dans la figure 3. Les valeurs de $I_{k}, \alpha$, et $\phi_{m}$ peuvent être déduites de ces courbes en utilisant l'une des trois méthodes suivantes :

(1) détermination de $I_{k}$ par une méthode graphique (Vollenweider 1965)

(2) régression linéaire entre les couples de valeurs de $P_{z}$ et $I_{z}$ pour $I_{z}<I_{k}$ (Platt \& Jassby 1976)

(3) calcul de $\phi_{\mathrm{m}}$, valeur moyenne de $\phi_{2}$ pour $\mathrm{I}<\mathrm{I}_{\mathrm{k}}$

La figure 4 montre que ces méthodes donnent des résultats très proches; les différences sont souvent liées aux profils divergents i.e. lorsque $P_{z}$ décroit plus vite que $I_{z}, \phi_{z}$ varie avec la profondeur. Il est possible que la diminution du rendement quantique ne soit qu'apparente puisque son calcul est basé sur l'hypothèse que $\epsilon_{E}$ ne varie pas avec la profondeur. La cou rbe (fig. 3) du 21.05 .87 mont re cependant que méme dans le cas de profils divergents, les méthodes ci-dessus sont utilisables.

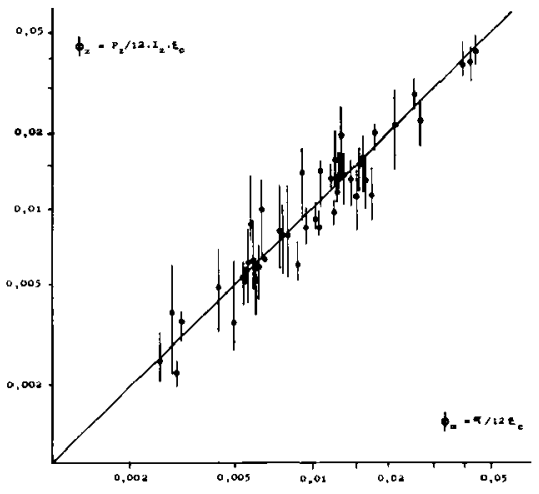

Fig. 4. Comparaison des valeurs du rendement quantique en lumière limitante estimées à partir des valeurs de $\alpha$ et calculées à partir de $\mathrm{I}_{\mathrm{z}}$ pour $\mathrm{I}_{\mathrm{z}}<\mathrm{I}_{\mathrm{k}}$; (amplitude et moyenne $\bullet$.

La figure 4 indique également que le rendement quantique aux intensités suboptimales d'éclairement varie dans les limites assez larges, sensiblement différentes de la plage de variation $(0,03$ à 0,06) donnée par Bannister (1974) et Bannister \& 
Tableau I. Amplitude, moyenne, écart type (s) et coefficient de variation (C.V.) des valeurs caractéristiques de la relation $\mathbf{P}$. I.

\begin{tabular}{|c|c|c|c|c|c|}
\hline & Unités & Amplitude & Moyenne & $\mathrm{s}$ & C.V. \\
\hline $\begin{array}{l}\mathrm{P}_{\text {opt }} \\
\alpha Q \\
\mathrm{I}_{\mathrm{k}} \\
\mathrm{I}_{\text {opt }} \\
\mathrm{I}_{\mathrm{opl}} / \mathrm{I}_{\mathrm{k}} \\
\phi_{\mathrm{m}}\end{array}$ & 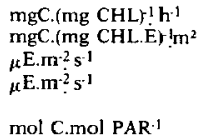 & $\begin{array}{c}0,234-6,56 \\
0,73-10,26 \\
90-280 \\
260-700 \\
1,61-5,77 \\
0,002-0,0425\end{array}$ & $\begin{array}{l}1,61 \\
2,85 \\
156 \\
435 \\
\quad 2,97 \\
0,0127\end{array}$ & $\begin{array}{l}1,368 \\
2,283 \\
46,5 \\
115 \\
\quad 0,91 \\
0,00936\end{array}$ & $\begin{array}{l}84 \% \\
79 \% \\
30 \% \\
27 \% \\
31 \% \\
74 \%\end{array}$ \\
\hline
\end{tabular}

Weidemann (1984). Cette variabilité affecte également les autres paramètres de la courbe $P=f(I)$ (Tableau I). Nos résultats indiquent que $I_{k}$ est le parametre le plus stable; sa valeur moyenne se situe dans la gamme des valeurs relevées dans la littérature (Harris, 1978 p. ex.). La plupart des travaux (Platt \& Jassby 1976, Harris 1978, Capblancq 1982, Coté \& Platt 1983. Tilzer 1984) donnent au contraire des valeurs de $\mathrm{P}_{\text {op! }}$ et de $\alpha$ plus élevées que celles qui figurent dans le tableau I. Ces dernières sont par contre tout à fait semblables à celles mesurées dans les lacs de l'ELA et du SAQ par Fee \& al. (1987). Compte tenu des différentes méthodes utilisées pour mesurer les taux de production, les concentrations de chlorophylle et pour estimer les paramètres, il est difficile de juger si ces différences sont liées à la nature du phytoplancton et aux propriétés du milieu.

Dans le cas d'une distribution verticale homogene des concentrations en chlorophylle, les paramètres estimés de la courbe $P=f(I)$ permettent de calculer la production intégrée sur la profondeur de la zone euphotique. La relation (Talling 1957, Vollenweider 1965) :

$$
\begin{gathered}
\Sigma A=C H L \cdot P_{\text {opt }} \cdot \epsilon \cdot \operatorname{Ln}\left(2 \mathrm{I}_{\alpha}^{\prime} / \mathrm{I}_{\mathrm{k}}\right)= \\
\text { CHL. } \mathbf{P}_{\text {op } 1} \cdot \epsilon \cdot \operatorname{Ln}\left(2 \alpha \mathrm{I}_{\mathrm{o}}^{\prime} / \mathbf{P}_{\mathrm{opt}}\right)
\end{gathered}
$$

donne des valeurs très proches de celles obtenues par intégration planimétrique des profis $A=f(Z)$ (fig. 5). Lorsque le peuplement phytoplanctonique est stratifié, l'intégration des profils de production ne peut se ramener à une équation simple.

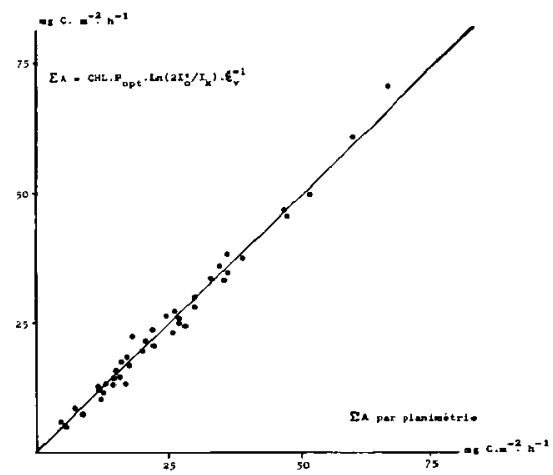

Fig. 5. Comparaison de la production photosynthétique intégrée sur la couche euphotique par planimétrie des profils verticaux et à l'aide de l'équation (5).

\section{Relations entre paramètres et composantes du milieu}

Les coefficients de corrélation entre les couples de valeurs de $P_{\text {opt }}, I_{k}$ et $\alpha-$ ce dernier étant par définition (équation 4 et fig. 4) proportionnel à $\phi_{\mathrm{m}}$ - indiquent l'existence d'une liaison particulièrement forte entre $P_{\text {opt }}$ et $\alpha$. Ces deux paramètres varient simultanément selon les équations de régression linéaire $(r=0,938 ; P<0,1 \%)$ :

$$
\alpha=0,35+1,6 \mathrm{P}_{\text {opt }}
$$




$$
\mathrm{P}_{\text {opt }}=0,55 \alpha \cdot 0,0064
$$

La covariation de ces deux paramètres (fig. 6) se traduit par une relative stabilité de $I_{k}$ dont les variations sont indépendantes de celles de $\mathrm{P}_{\text {opt }}(\mathrm{r}=$ $0,164)$ et de $\alpha(r=-0,117)$. Nos résultats indiquent par ailleurs que la relation entre $P_{\text {opt }}$ et $\alpha$ ne varie pas de façon significative au cours des saisons.

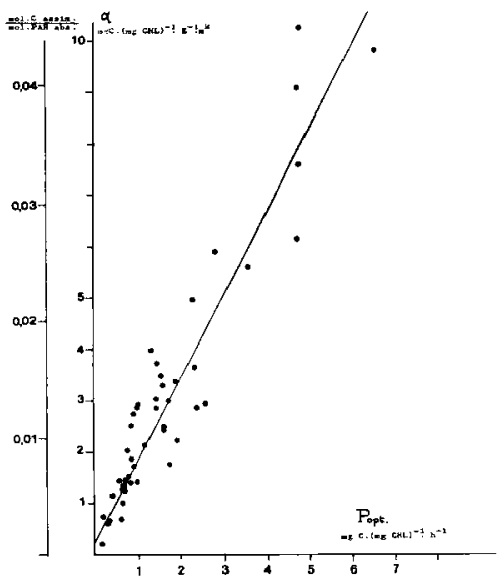

Fig. 6. Relation entre $P_{\text {opt }}$ et $\alpha$. La correspondance entre $\alpha$ et $\Phi_{m}$ est calculée à partir de l'équation (4) avec $\epsilon_{c}=$ $0,02 \mathrm{mg}^{-1}$ CHL.m².

La relation empirique qui lie $\mathrm{P}_{\text {opt }}$ et $\alpha$ suggère que ces deux paramètres dépendent des mêmes facteurs. On admet généralement que la température n'agit pas sur $\alpha$ mais qu'elle contrôle, via l'activité enzymatique, le paramètre $P_{\text {opt }}$. Plusieurs travaux (cités in Harris 1978, Capblancq 1982, Coté \& Platt 1983 ) indiquent par contre que la préadaptation des algues à des éclairements plus ou moins intenses, les conditions nutritives, la taille des cellules ou la composition spécifique du peuplement peuvent agir sur ces deux paramètres. L'effet de ces facteurs sur la réponse photosynthétique des communautés naturelles est cependant difficile à discerner d'une part, du fait de leur variation simultanée, d'autre part parce que ces communautés évoluent dans un environnement turbulent.
Tableau II. Matrice de corrélations entre $\mathbf{P}_{\text {opt, tempéra- }}$ ture, stabilité thermique de la couche euphotique (S), éclairement moyen pendant les trois jours précédents ( $\mathrm{E}=$ durée du jour $\mathrm{x}$ intensité moyenne) et concentra. tions en chlorophylle; (valeurs significatives au seuil $0,1 \%{ }^{* *}, 1 \%{ }^{*}$ et $5 \%+$ ).

\begin{tabular}{|c|c|c|c|c|c|c|}
\hline & $P_{\text {op } 1}$ & & Temp. & $s$ & $E$ & $\mathrm{CHL}$ \\
\hline $\begin{array}{l}\mathrm{P}_{\text {opt }} \\
\alpha \\
\text { Temp. } \\
\mathrm{S} \\
\mathrm{E} \\
\mathrm{CHL}\end{array}$ & 1 & $\begin{array}{l}0,938^{* * *} \\
1\end{array}$ & $\begin{array}{l}{ }^{\star} 0,545^{\star \star \star} \\
0,476^{\star \star \star} \\
1\end{array}$ & $\begin{array}{l}0,245 \\
0,182 \\
0,604^{\star \star} \\
1\end{array}$ & $\begin{array}{l}0,426^{*} \\
0,471^{* *} \\
{ }^{*} 0,482^{* *} \\
0,422^{*} \\
1\end{array}$ & $\begin{array}{l}-0,627^{* *} \\
-0,602^{* *} \\
-0,621^{* *} \\
-0,440^{*} \\
-0,366+ \\
1\end{array}$ \\
\hline
\end{tabular}

La matrice de corrélations du tableau II indique une liaison apparente avec la température ; l'équation de régression $\left(P_{\text {opt }}=0,425.1,078^{\mathrm{T}}\right)$ correspond à un $Q_{10}=2,02$, valeur généralement admise. Cependant, à l'évolution saisonnière de la température sont liés:

(1) l'évolution de la biomasse de phytoplancton ; nos résultats montrent que $\mathrm{P}_{\text {opt }}$ diminue selon une fonction puissance de la concentration en chlorophylle $\left(\mathrm{P}_{\mathrm{opt}}=2,87 . \mathrm{CHL}-0,64\right)$. L'analy se des corrélations partielles (Tableau III) d'un modèle de régression multiple entre $\mathrm{P}_{\text {opt }}, \mathrm{T}$ et $\mathrm{CHL}\left(\mathrm{R}^{2}=0,425 ; \mathrm{DL}\right.$ $=45$ ) indique que la corrélation entre $\mathrm{P}_{\mathrm{opt}}$ et la quantité de chlorophylle (i.e. la biomasse de phytoplancton) est indépendante de la température. $\mathrm{Ce}$ résultat rejoint les conclusions de Fee \& al. (1987) selon lesquelles la température détermine uniquement la limite supérieure de $\mathbf{P}_{\mathrm{opt}}$ -

(2) les changements de la composition du peuplement: les plus faibles températures et les maximums de chlorophylle correspondent au peuplement printanier à base de Diatomées. A partir du mois de juin, ces dernières représentent moins de $25 \%$ de la biomasse; les variations de $\mathrm{P}_{\text {opt }}$ sont alors corrélées $(r=0,602 ; P<1 \%)$ au pourcentage de biomasse formé par les Pyrrophytes.

(3) la stabilité thermique de la couche euphotique (S) et l'éclairement incident ( $\mathrm{E}=$ durée du jour $\mathrm{x}$ intensité moyenne). La valeur moyenne de $\mathrm{E}$ au cours des trois jours précédant les mesures est corrélée à l'ensemble des variables considérées; il n'apparaît par contre aucune relation entre $\mathrm{S}, \mathrm{P}_{\text {opt }}$ et $\alpha$ (Tableau III). 


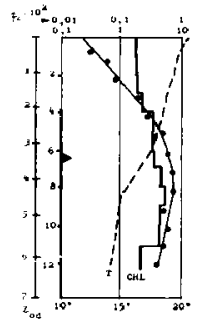

30.05 .87

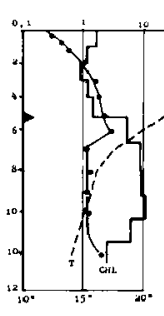

$16.07+87$

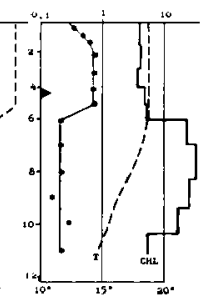

22.07 .87

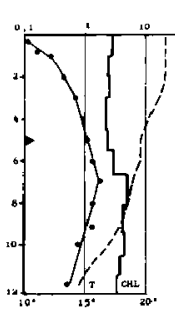

10.08.87?

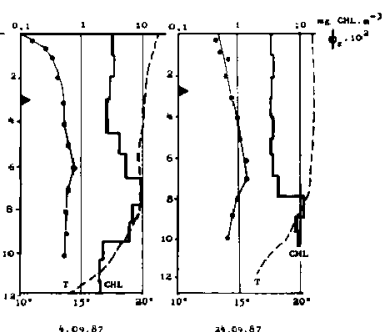

0.09 .87

Fig. 7. Profils verticaux de temperature (-), de concent ration en chlorophylle (histogrammes) et du rendement quantique global de la photosynthèse (-๑) durant l'été 1987. La profondeur est portée sur une échelle graduée en unités de $Z_{0 d}$ (figurée à gauche); la flèche indique la profondeur correspondant à une intensité de $150 \mu$ E. m$^{2} \mathrm{~s}^{-1}$, valeur moyenne de $I_{k}$.

Tableau III. Cuefficients de corrélation totale (en bas, à gauche) et partielle (en haut, à droite) entre $\mathrm{P}_{\mathrm{opt}}$, la tempé rature et la quantité de chlorophylle ${ }^{* *}=$ valeurs significatives à $P(0,01$ ).

\begin{tabular}{llll}
\hline & $\mathbf{P}_{\text {opt }}$ & Temp. & CHL \\
\hline$P_{\text {opt }}$ & 1 & 0,235 & $-0,439^{* *}$ \\
Temp. & $0,545^{* *}$ & 1 & $-0,428^{* *}$ \\
CHL & $-0.627^{* *}$ & $-0,621^{* *}$ & 1 \\
\hline
\end{tabular}

Les profils verticaux du rendement quantique de la photosynthèse ( $\mathrm{fig}$. 7) confirment cependant que la ségrégation des algues au sein d'une colonne d'eau stratifiée modifie leur réponse à la lumière. Les conséquences d'une stratification thermique prolongée apparaissent en particulier dans l'évolution des profils entre le 30.06 et le 22.07 où l'on voit se différencier, au niveau de la thermocline, un peuplement adapté aux faibles éclairements qui se caractérise par un rendement quantique amoindri par rapport à celui du peuplement de l'épilimnion. Nos mesures ne permettent pas toutefois d'affirmer que la diminution de $\phi$ est réelle puisque $\epsilon_{\mathrm{c}}$ peut varier en profondeur en fonction de la composition spectrale de la lumière transmise, des changements d'espèces et de l'état physiologique des algues.

\section{Discussion et conclusion}

Il apparaît de plus en plus évident que les valeurs caractéristiques $\left(P_{\text {opt }}, \alpha, \phi_{m}\right)$ de la relation $P=f(I)$ varient au cours de l'année (Platt \& Jassby 1976, nos résultats), d'un lac à l'autre (Fee \& al. 1987) d'un jour à l'autre (Coté \& Platt 1983) et au cours d'une mème journée (Harding \& al. 1982). La variabilité de ces paramètres apparaît done comme une propriété inhérente au phytoplancton.

Quelques travaux récents (Harris 1984, Falkowski 1984) soulignent en effet la très grande plasticité des algues planctoniques. Ces organismes peuvent répondre aux changements de leur environnement climatique et chimique sur des échelles de temps variant de l'heure à quelques semaines par une variété d'adaptations. Ces réponses adaptatives qui se traduisent par des al térations de la structure des chloroplastes et du métabolisme, des modifications de la composition chimique (p. ex. rapports $\mathrm{C}: \mathrm{N}: \mathrm{CHL}$ ), des variations du volume des cellules et, enfin, par des remplacements d'especes affectent l'activité photosynthétique. Dans les milieux naturels, cette séquence d'adaptation est fréquemment perturbée par les fluctuations de l'éclairement (liées à la succession de jours clairs et nuageux et à l'intensité du mélange turbulent) qui interfèrent avec le cycle saisonnier d'évolution du milieu. Les variations hebdomadaires des taux de photosynthèse 
spécifique apparaissent ainsi comme résultant à la fois des ajustements du métabolisme aux conditions d'éclairement de quelquues heures précédant la mesure et des changements plus lents de la structure du phytoplancton qui intervienment à l'échelle de la semaine.

L'interférence de ces effets explique probablement la forte dispersion des valeurs par rapport à la courbe moyenne d'évolution de $P_{\text {opt }}$ et de $\alpha$ au cours d'un cycle annuel. Cet te évolution annuelle est inversement corrélée à celle de la biomasse de phytoplancton. Une relation inverse avec la biomasse apparaît également dans les profils de l'été 1987. La diminution du taux de photosynthèse spécifique lorsque la biomasse (exprimée en biovolume ou en chlorophylle) augmente a été mentionnée par de nombreux auteurs et interprétée comme le reflet d'une compétition vis-à-vis des éléments nutritifs (cf. Javornicky in Westlake 1980 p. ex.). Les variations de biomasse au cours d'une année sont cependant liées à celles de nombreux autres facteurs susceptibles d'agir directement sur l'activité photosynthétique.

Le point le plus important qui ressort de nos résultats est la variation simultanée de $\mathrm{P}_{\text {opt }}$ et de $\alpha$, deux paramètres souvent considérés comme indépendants. La covariation de ces deux valeurs, qui remet en cause 'hypothèse d'une relative stabilité du produit $\epsilon_{\mathrm{C}}, \phi_{\mathrm{I}}$ (Bannister \& Weidemann 1984), apparaît également dans les relations établies à partir de séries de mesures effectuées en milieu marin (Harding \& al. 1982, Coté \& Platt 1983). Contrairement à Coté \& Platt qui trouvent que la relation entre $P_{\text {opt }}$ et $\alpha$ varie au cours de leur période d'étude (70 jours) nos résultats ne révèlent aucune tendance saisonnière dans la relation entre ces deux paramètres.

L'intérêt de ces relations est de permettre l'estimation de $\alpha$ (et de $I_{k}$ ) à partir de la seule déterminaton de $\mathrm{P}_{\mathrm{opt}}$. Cette dernière étant plus simple à réaliser qu'un profil complet de mesures sur toute la colonne d'eau, on peut envisager la possibilité de mesures plus fréquentes de $P_{\text {opt }}$ combinées avec des déterminations plus espacées de $\alpha$ comme un moyen de réduire les incertitudes liées à la variabi. lité de l'activité photosyrathétique du phytoplancton.

La variabilité des paramètres de la relation $\mathbf{P}=$ $\mathrm{f}(\mathrm{I})$ est la principale limite à l'utilisation des modèles de production primaire pour convertir les taux de production instantannés en taux journalier, pour extrapoler les valeurs entre deux mesures successives ou pour estimer la production à partir des seules mesures de chlorophyl le et de lumière. Le développement de ces modèles est donc lié à notre capacité à prévoir les valeurs de ces paramètres. La principale difficulté provient du fait que ces valeurs sont une fonction complexe du temps i.e. que la réponse photosynthétique du phytoplancton à la lumière de l'instant $t$ dépend des conditions climatiques et chimiques de la "période précédente ". Les questions qui se posent alors concernent la définition de ces conditions dans un environnement turbulent et de l'échelle de temps à prendre en compte. Les tentatives de simulation de la réponse photosynthétique du phytoplancton aux fluctuations de la lumière engendrées par le mélange turbulent ne portent pour l'instant que sur des échelles de temps de l'ordre de la journée (Falkowski 1984). La modélisation des mécanismes qui opèrent sur des échelles de temps plus longues est beaucoup plus difficilement concevable.

\section{Remerciements}

Cette étude a été réalisée dans le cadre du GRECOCNRS "Fonctionnement des systèmes lacustres " et d'un programme de recherches sur la retenue de Pareloup subventionnée par le Ministère de l'Environnement. Nous remercions la Direction des Etudes et Recherches d'EDF qui nous a permis d'utiliser la base technique de Pareloup ainsi que MM. Tourenq, Lavandier, Le Cohu et Dalger pour l'aide apportee sur le terrain.

\section{Travaux cités}

Bannister (T.T.). 1974. - Production equations in terms of chlo rophyll concentration, quantum yield and upper limit to pro duction. Limnol. Oceanogr., 19:1-12.

Bannister (T.T.) \& Weidemann (A.D.). 1984. - The maximum quantum yield of phytoplankton photosynthesis in situ. $J$. Planktun Res., 6 : 275-294.

Capblancq (J.). 1982. - Phytoplancton et production primaire. in "Écologie du plancton des eaux continentales ". Collection Ecologie. 16, Masson, Paris, 198 p.

Côté (B.) \& Platt (T.). 1983. - Day to day variations in the spring summer photosynthetic parameters of coastal marine phytoplankton. Limnol. Oceanogr., $28: 320-344$.

Falkowski (P.G.). 1984. - Physiological res ponses of phytoplank ton to natural light regimes. J. Plankton Res., 6: 295-307.

Fee (E.J.), Hecky (R.E.) \& Welch (H.A.). 1987. - Phytoplankton photosynthesis parameters in central Canadian lakes. J. Plankion Res., $9:$ 305-316.

Harding (L.W.), Prezelin (B.B.), Sweeney (B.M.) \& Cox (J.L.) 1982. - Diel oscillations of photosynthesis-irradiance (P-I) relationship in natural assemblages of phytoplankton. Mar. Biol., 67 : 167-178.

Harris (G.P.). 1978. - Photosynthesis, production and growth : the physiological ecology of phytoplankton. Ergeb. Limnol., 10: 1-171. 
Harris (G.P.). 1984. - Phytoplankton productivity and growth measurements : past, present and future. J. Plankton Res., 6 : 219-237.

Jassby (A.) \& Platt (T.). 1976. - Mathematical formulation of the relationship between photosynthesis and light for phytoplank ton. Limmol. Oceanogr., $21: 540-547$.

Marker (A.F.H.I. Nush (E.A.) \& Riemann (B.). 1980. - The measurements of photosynthetic pigments in freshwater and standardization of methods: conclusions and considerations. Ergeb. Limnol, $14: 91-106$.

Plat1 (T.) \& Jassby (A.D.). 1976. - The relationship between photosynthesis and light for natural assemblages of coastal marine phytoplankton. J. Phycol. $12: 421430$.

Rodhe (W.). 1965. - Standard correlations between pelagic photosynthesis and light. Mem. Ist. Ital. Idrobiol, 18 (suppl): $367-381$.
Talling (J.F.). 1957. - The phytoplancton population as a compound photosynthetic system. New: Phytol, $56: 133-149$.

Tilzer (M.). 1984 - The quantum yield as a fundanental parameter controlling vertical photosynthetic profites of phytoplankton in lake Constance. Arch. Hydrobiol/suppl. 69: 169-198.

Vollenweider (R.A.). 1965. - Calculation models of photosynthesis-depth curves and some implications regarding day rate estimates in primary production measurements. Mem lst. Irol. Idrobiol., 18 (suppl.) 425.457.

Westlake (D.F.). 1980. - Primary production in Le Cren (E.D.) \& Lowe-Mc Connel (R.H.), eds. : The functionning of freshwater ecosystems ». IBP 22, Cambridge Univ, Press, 588 p. 